\title{
As pesquisas etnodirigidas na descoberta de novos fármacos de interesse médico e farmacêutico: fragilidades e pespectivas
}

\author{
Ulysses Paulino de Albuquerque ${ }^{1 *}$, Natália Hanazaki ${ }^{2}$ \\ ${ }^{1}$ Universidade Federal Rural de Pernambuco, Departamento de Biologia, Laboratório de Etnobotânica Aplicada, \\ 52171-030, Recife, PE, Brasil, \\ ${ }^{2}$ Universidade Federal de Santa Catarina, Departamento de Ecologia e Zoologia, Laboratório de Etnobotânica e \\ Ecologia Humana, 88040-900, Florianópolis, SC, Brasil,
}

\begin{abstract}
RESUMO: As investigações etnofarmacológicas e etnobotânicas têm sido a principal abordagem reconhecida por cientistas em todo o mundo, como uma estratégia de seleção de plantas medicinais. As qualidades e fortalezas dessas abordagens já foram suficientemente discutidas, restando poucas dúvidas de seu potencial e impactos biológicos, econômicos e sociais. Este artigo focaliza os estudos etnodirigidos (etnobotânicos e etnofarmacológicos) levantando os problemas encontrados na qualidade das pesquisas, que podem afetar a sua aplicação na descoberta de novos fármacos de interesse médico e farmacêutico. Adicionalmente, apontamos alguns elementos que podem colaborar para a melhoria da qualidade das abordagens e publicações e que podem ser aplicados a diferentes estudos etnodirigidos. Nós também sugerimos que os investigadores reconsiderem algumas das abordagens dominantes, bem como a visão, não raro simplista, desses estudos.
\end{abstract}

Unitermos: Etnobotânica, etnofarmacologia, métodos em etnobotânica, método científico, plantas medicinais, bioprospecção.

\begin{abstract}
Ethnodirected research in the discovery of new drugs of medical and pharmaceutical interest: flaws and perspectives". Ethnopharmacological and ethnobotanical investigations have been the main approach used by scientists all over the world as a strategy to select medicinal plants. The qualities and strengths of these approaches have been sufficiently discussed, and there are a few doubts left in relation to their potential and biological, economic, and social impacts. This article focuses on ethnodirected studies (ethnopharmacological and ethnobotanical), raising problems found in the quality of these studies. In addition, we point out some elements that might contribute to the improvement of approaches and publications that can be applied to different ethnodirected studies. We also suggest that researchers reconsider some of the dominant approaches, as well as the view of these studies, which is often simplistic..
\end{abstract}

Keywords: Ethnobotany, ethnopharmacology, methods in ethnobotany, scientific methods, medicinal plants, bioprospecting.

\section{INTRODUÇÃO}

Muitas áreas estão envolvidas na pesquisa de novas substâncias oriundas de plantas, como a fitoquímica, que trabalha no isolamento, purificação e caracterização de princípios ativos; a etnobotânica e a etnofarmacologia, que buscam informações a partir do conhecimento de diferentes povos e etnias; e a farmacologia, que estuda os efeitos farmacológicos de extratos e dos constituintes químicos isolados (Maciel et al., 2002; Mendonça-Filho; Menezes, 2003; Vendruscolo et al., 2005). Nos últimos anos, no Brasil, várias pesquisas foram realizadas com contribuições relevantes sobre o assunto, não só quanto a aspectos fitoquímicos, mas também quanto à atividade biológica de plantas que ocorrem nos diferentes ecossistemas brasileiros (Desmarchelier et al., 1999; Jorge et al., 2004; Duarte et al., 2004; Lima et al., 2006;
Hiruma-Lima et al., 2006).

Há, nesse sentido, vários caminhos para o estudo de plantas medicinais, destacando-se quatro tipos básicos de abordagens: randômica, etológica, quimiotaxonômica e etnodirigida. As investigações randômicas compreendem a coleta ao acaso de plantas para triagens fitoquímicas e farmacológicas. Há muitas críticas e visões equivocadas sobre esta abordagem, devido a aleatoriedade, o que não implica em ausência de critérios. Pesquisadores no Panamá (Calderón et al., 2000) utilizaram um desdobramento deste procedimento para estudar as plantas de um bosque tropical, partindo de uma lista de plantas já estudadas em 50 ha. Na pesquisa, estes autores tiveram o cuidado de estudar a lista de plantas inventariadas na área, e selecionar as espécies com necessidade de serem investigadas por meio do conhecimento prévio, reportado na literatura. Com isto, uma nova lista de espécies foi 
criada e os resultados foram bastante satisfatórios, uma vez que se identificou uma boa diversidade de plantas com atividades anti-oxidante e anti-tumoral.

A abordagem quimiotaxonômica ou filogenética consiste na seleção de espécies de uma família ou gênero, para as quais se tenha algum conhecimento fitoquímico de ao menos uma espécie do grupo. Um bom exemplo são as espécies do gênero Bauhinia que possuem algumas substâncias químicas em comum como glicosídeos, triterpenos, lactonas e flavonóides (Silva; Cechinel Filho, 2002). Outro exemplo são as espécies do gênero Hypericum, notadamente $H$. perforatum L. Hipericina é um dos principais constituintes desta espécie, ao qual se reportam propriedades antidepressivas. Em $H$. barbatum Jacq. encontrou-se conteúdo de hipericina 3.9 vezes maior que em $H$. perforatum (Smelcerovic; Spiteller, 2006). Entre as espécies brasileiras estudadas, H. caprifoliatum Cham. \& Schltdl. também mostrou atividade antidepressiva (Daudt et al., 2000).

Um caminho recentemente apontado para a descoberta de novos fármacos é baseado nos estudos de comportamento animal com primatas (Krief et al., 2004), ou abordagem etológica. Esta abordagem tem como orientação avaliar a utilização de metabólitos secundários por animais, ou outras substâncias não nutricionais dos vegetais, com a finalidade de combater doenças ou controlá-las (Huffman, 2003; Carrai et al., 2003). Evidências a partir do uso de plantas por grandes símios africanos, no combate a infecções parasitárias, sinalizam positivamente para esse nova rota de investigação. Alguns exemplos podem ser esclarecedores.

Fêmeas de lêmures (Propithecus verreauxi) ingerem plantas ricas em taninos no período de lactação, sugerindo que o consumo está associado com o aumento no peso corpóreo e estimulação da secreção do leite e, tendo em vista as propriedades biológicas desses polifenóis, o aumento da ingestão de taninos pode ser um comportamento profilático de auto-medicação (Carrai et al., 2003). Folhas de Trichilia rubescens Oliv., consumidas por chimpanzés de Uganda, revelaram atividade antimalárica in vitro suportando a hipótese de que a dieta de chimpanzés contém metabólitos secundários de interesse médico (Krief et al., 2004). Entretanto, uma das deficiências na interpretação da automedicação por animais reside em saber se a ingestão é intencional ou secundariamente como resultado do simples ato de se alimentar (Huffman, 2001, 2003). Além disso, a obtenção de informações pode ser um processo lento e exaustivo, uma vez que implica em acompanhar o comportamento de um grupo de animais e extrair, dessas observações, o que possa interessar a um estudo farmacológico.

Finalmente, a abordagem etnodirigida consiste na seleção de espécies de acordo com a indicação de grupos populacionais específicos em determinados contextos de uso, enfatizando a busca pelo conhecimento construído localmente a respeito de seus recursos naturais e a aplicação que fazem deles em seus sistemas de saúde e doença. Este é um dos caminhos utilizados atualmente, por duas razões básicas: o tempo e o baixo custo envolvidos na coleta dessas informações (Maciel et al., 2002). Duas disciplinas científicas têm se destacado nesta tarefa: a etnobotânica e a etnofarmacologia. Por definição, a etnobotânica se ocupa da "inter-relação direta entre pessoas e plantas" (Albuquerque, 2005), incluindo todas as formas de percepção e apropriação dos recursos vegetais; e a etnofarmacologia se ocupa do estudo dos preparados tradicionais utilizados em sistemas de saúde e doença que incluem isoladamente ou em conjunto plantas, animais, fungos ou minerais. Ampliando a análise dessa definição, uma das visões para a etnofarmacologia defende que seu objetivo é avaliar a eficácia das técnicas "tradicionais" fazendo uso de um grande número de modelos farmacológicos (Waller, 1993). Há várias definições para etnofarmacologia, mas, a título de ilustração, Di-Stasi (2005) a entende como "a identificação e o registro dos diferentes usos medicinais de plantas por diferentes grupos". Esta é uma definição que se distancia um pouco da clássica noção de observação, descrição e investigação experimental das drogas indígenas e suas atividades biológicas (Bruhn; Holmstead, 1980), uma vez que nesta última o foco da etnofarmacologia recai sobre todos os recursos que essas culturas fazem uso (não só plantas), bem como indo mais além do que o registro dessas drogas, mas incluindo uma avaliação experimental.

Desde a publicação de trabalhos, principalmente a partir da década de 1990, sobre o potencial de tais investigações para descoberta de novos fármacos, a pesquisa envolvendo os saberes e práticas "tradicionais" ganharam novo sentido indo além da simples compilação de plantas e animais (ver, por exemplo, Khafagi; Dewedar, 2000; Morais et al., 2005; Monteiro et al., 2006; Albuquerque, 2006a; Albuquerque et al., 2007). Sob a suposição tácita de que o sucesso na descoberta de novos fármacos seria maior a partir de pesquisas etnodirigidas, houve um incremento significativo em tais pesquisas. Alguns exemplos podem justificar essa tendência. Em testes de extratos anti-HIV, uma pequena amostra de plantas oriundas de um curandeiro de uma vila em Belize, na América Central, apresentou-se quatro vezes mais eficiente do que a triagem realizada com uma coleção aleatória (Balick; Cox, 1996). Bioensaios in vitro comprovaram a atividade biológica sugerida por curandeiros indígenas em $74 \%$ das vezes (Balick; Cox, 1996). Estudos comparativos entre o método etnodirigido e o aleatório vêm demonstrando a superioridade do primeiro em diferentes situações (ver tabelas 1 e 2 ). Slish et al. (1999) compararam o percentual de atividade de plantas coletadas aleatoriamente contra as coletadas por meio do método etnodirigido, mensurada através de contrações induzidas em músculo liso, encontrando que para o estudo etnobotânico o efeito observado foi de $12,9 \%$ do total de espécies $(n=31)$ ou $13 \%$ do total de amostras $(n=46)$, enquanto que para o estudo aleatório 
Tabela 1. Comparação do percentual de atividade (\%) antimicrobiana de plantas oriundas da Península do Sinai (Egito) coletadas aleatoriamente contra as coletadas por meio do método etnodirigido. Reproduzido de Khafagi; Dewedar (2000).

\begin{tabular}{lcc}
\hline \multicolumn{1}{c}{ Parâmetro } & \multicolumn{2}{c}{ Método Coleta } \\
\hline & Aleatório & Etnodirigido \\
\hline Total de espécies testadas & 36 & 24 \\
Percentual ativo & $41,7^{\mathrm{a}}$ & $83,3^{\mathrm{a}}$ \\
Forte atividade & 13,9 & 8,3 \\
Moderada & 13,9 & 50 \\
Fraca & 13,9 & 25 \\
\hline
\end{tabular}

aDiferenças significativas pelo teste de Fisher

Tabela 2. Atividade larvicida contra Haemonchus contortus de plantas medicinais da Costa do Marfim (África) selecionadas com base em critérios etnobotânicos. Fonte: Diehl et al. (2004).

\begin{tabular}{lcc}
\hline & Total & Altamente ativo \\
\hline Espécies testadas & 60 & $31,7 \%$ \\
Extratos testados & 86 & $25,6 \%$ \\
\hline
\end{tabular}

nenhum efeito foi observado para 32 espécies e 44 amostras. Em outros estudos, mesmo sem pretensões comparativas, os resultados de pesquisas etnodirigidas são entusiasmadores (ver tabela 2).

Tanto a etnobotânica como a etnofarmacologia têm demonstrado ser poderosas ferramentas na busca por substâncias naturais de ação terapêutica. Apesar disso, alguns limitantes a tais abordagens podem ser mencionados, como: a dificuldade de coletar informações fidedignas das pessoas; o fato do uso de plantas em diferentes culturas encontrar-se sempre associado, em maior ou menor grau, a componentes mágico-religiosos; a existência de questões éticas que envolvem acesso a conhecimento tradicional associado ao uso da biodiversidade. Sem dúvida, muitos estudos ditos etnodirigidos têm sido desenvolvidos à margem dessas discussões, o que tem levado a várias situações: publicações com problemas éticos, metodológicos, teóricos, com resultados pobres e limitados quanto à sua aplicação utilitária para a descoberta de novos fármacos. Apesar dessa situação, alguns estudos desenvolvidos (como os relatados anteriormente) sugerem que a informação sobre novos fármacos de interesse médico e farmacêutico pode ser obtida de forma mais efetiva estudando as práticas terapêuticas de diferentes povos e culturas. Assim, a proposta deste artigo é de revisar alguns aspectos importantes para garantir a coleta de informações confiáveis em uma pesquisa etnodirigida para a descoberta de novos fármacos, apresentando uma síntese dos elementos que vêm sendo considerados indispensáveis para garantir a qualidade das publicações. Para tanto, é importante esclarecer alguns dos termos e conceitos adotados neste artigo. Quando falarmos de "etnodirigido", estaremos nos referindo aos estudos etnobotânicos e/ou etnofarmacológicos que visam contribuir com a descoberta de novos fármacos de interesse médico ou farmacêutico, ou que tenham potencial de aplicação nesse setor. $O$ termo tradicional apresenta diferentes entendimentos (ver, por exemplo, Alves; Albuquerque, 2005; Diegues; Arruda, 2001), mas aqui será aplicado em dois contextos: comunidades tradicionais e saber ou conhecimento tradicional (tomando como referência comunidades tradicionais). Alternativamente a esse termo tem sido proposto o termo conhecimento local, como o conjunto de "conhecimentos, práticas e crenças, desenvolvidos por processos adaptativos e passados entre gerações por transmissão cultural, sobre as relações entre os seres vivos entre si e com o seu ambiente" (Gadgil et al., 1993; Berkes, 1999). Vale destacar que nem todo estudo etnobotânico sobre plantas usadas tradicionalmente é dirigido, a priori, para contribuir com a descoberta de novos fármacos de interesse médico ou farmacêutico.

A grande contribuição de muitos estudos é apresentar uma lista de espécies (animais ou plantas) com informações sobre os seus usos por comunidades locais (ver bons exemplos em Almeida; Albuquerque, 2002; Nunes et al., 2003; Macedo; Ferreira, 2004; Gazzaneo et al., 2005; Alves; Rosa, 2006). Algumas dessas abordagens, sendo essencialmente descritivas, continuam resgatando o período inicial das investigações etnocientíficas, fortemente carregadas de uma influência etnográfica (ver Albuquerque, 2005). Todavia, vários estudos, apesar de não terem o objetivo inicial de contribuir com a descoberta de novos fármacos, mostram potencial de desdobramento no sentido de fortalecer essa área de pesquisa. A maioria das contribuições, no entanto, não sinaliza ou se direciona no sentido de resolver algumas questões básicas. Por exemplo: como as pessoas selecionam plantas e animais para uso medicinal? Quais os critérios envolvidos nessas escolhas? Essas 
Quadro 1. Proposta de estruturação de artigos científicos em etnobotânica e etnofarmacologia. Modificado de McClatchey (2006).

$1^{\mathrm{a}}$. Etapa

1. Evite começar escrevendo o título, resumo, introdução ou a revisão de literatura. Deixe tudo isso para o final da redação do artigo.

2. Comece escrevendo as hipóteses e/ou os objetivos da pesquisa. Seja o mais claro possível. Prepare uma lista de referências relacionadas às suas hipóteses e/ou objetivos.

3. Caracterize precisamente a área de estudo, a população estudada e o universo amostral.

4. Descreva criteriosamente os métodos usados, relacionando-os com as suas hipóteses e/ou seus objetivos. Verifique se os mesmos métodos foram usados e/ou descritos por outros autores e cite-os adequadamente. Algumas vezes é interessante explicitar qual foi o contexto de aplicação desses métodos por outros pesquisadores. Se usar novos métodos, descreva-os detalhada e claramente.

5. Seja claro sobre os métodos usados. Quando possível argumente sobre a sua escolha dos métodos, seu potencial e suas limitações.

$2^{\text {a. }}$ Etapa

6. Organize os resultados com base nos métodos usados. Organize tabelas, figuras, e descrições etnográficas dos resultados. Análises estatísticas dos resultados devem ser incluídas e claramente referenciadas.

7. Escreva os resultados de uma forma simples usando as sentenças para descrever as relações entre os métodos usados, tabelas e figuras dos resultados. Em muitos casos, isso consiste de uma frase simples e pequena. Prepare uma lista de referências que ajudará a discutir os seus resultados.

$3^{\mathrm{a}}$. Etapa

8. Interprete os resultados a luz das hipóteses e objetivos. Faça uma lista de referências relacionadas com a sua pesquisa.

9. Escreva as referências completas das citações que você usa. É muito fácil esquecer depois o material que você usou.

10. Escreva discussões claras dos resultados. A primeira discussão deve estar na ordem de sua apresentação dos resultados. Não repita dados dos resultados na discussão.

11. Avalie claramente e especificamente as suas hipóteses, indicando como os seus resultados suportam-nas ou rejeitam-nas.

\section{$4^{\mathrm{a}}$. Etapa}

12. Quando hipóteses são usadas com bons métodos, teorias gerais sobre a relação pessoas/plantas podem surgir. Organize os parágrafos em uma ordem lógica.

13. Resumo. Escreva uma ou duas frases que sintetizem a introdução da pesquisa. Com uma frase apresente as hipóteses ou objetivos. Escreva uma ou duas frases que resumam os métodos. Escreva uma ou duas frases que sintetizem a discussão dos resultados. E uma ou duas frases para as conclusões.

14. Crie um título que seja descritivo da pesquisa que foi feita. Alguns autores têm adotado títulos na forma de perguntas que indicam para o leitor o teor da publicação. Outras vezes, usam-se frases afirmativas, por exemplo: "A distância dos centros urbanos afeta o conhecimento sobre plantas medicinais dos indios Piaroa".

15. Prepare uma lista de palavras-chaves. Não repita palavras do título.

16. Solicite a um colega, não envolvido diretamente com a pesquisa, que faça uma leitura crítica de seu material. Entregue a ele, caso não disponha, uma cópia deste roteiro.

17. Não se esqueça de conferir a padronização bibliográfica e a integralidade das referências utilizadas no texto e citadas na lista de referências.

questões não estão resolvidas, embora há estudos que já começam sinalizar para a existência de padrões (Steep; Moerman, 2001; Steep, 2004; Voeks, 2004; Almeida et al., 2005; Albuquerque; Lucena, 2005; Monteiro et al., 2006; Estomba et al., 2006; Albuquerque, 2006a) que, se confirmados, nos possibilitarão prever que grupos de plantas são potenciais candidatos para apresentarem atividade biológica interessante e significativa. Um desses possíveis padrões diz respeito à "preferência" das pessoas por selecionarem plantas de ambientes "perturbados" para uso como medicinais (ver Voeks, 2004). Nesse sentido, nós propomos que os investigadores procurem associar o tradicional levantamento etnobotânico com testes de hipóteses (ver Albuquerque; Lucena, 2005).

\section{Principais fragilidades dos trabalhos publicados com relação à descoberta de novos fármacos}

A identificação de problemas de pesquisa centrais para o avanço da área e a melhoria na qualidade 
das publicações só é possível com o uso da literatura científica que reflete todo o conhecimento previamente construído sobre o assunto. Este é um dos motivos que resulta na pobre qualidade dos trabalhos. Referindo-se aos trabalhos em etnobotânica de maneira geral, McClatchey (2006) ressalta que a pobre qualidade na apresentação da ciência indica que faltaram aos autores: habilidade na redação, fluência no idioma usado, modelos positivos de redação de qualidade, bem como acesso a métodos básicos de pesquisa. Por um lado, a avaliação de algumas das publicações no Brasil parece sustentar a opinião de McClatchey, de outro lado os pesquisadores brasileiros têm hoje acesso facilitado à literatura científica mundial, o que não explica a ausência de rigor na coleta e análise dos dados.

Muitos problemas, que não raro são a causa de rejeição de artigos em periódicos com corpo especializado de revisores, estão relacionados à compatibilidade dos dados com a teoria ou hipóteses testadas. Nós sugerimos que os trabalhos apresentem uma descrição clara e detalhada dos métodos, principalmente daqueles que representam novas abordagens e a citação de literatura da referência para esses métodos. No quadro 1 nós apresentamos uma proposta para estruturação de artigos com abordagens etnodirigidas, no escopo da etnobotânica/ etnofarmacologia.

Alguns elementos são de presença obrigatória em estudos etnodirigidos e, no quadro 2, apresentamos um roteiro simples, como proposta básica de avaliação de publicações, tomando por base o que usualmente é aceito para as abordagens científicas que envolvem etnobotânica e etnofarmacologia. Se as perguntas são as principais diretrizes de uma pesquisa, os métodos adequados permitem a melhor forma de medir um fenômeno, tirando conclusões consubstanciadas desses achados. Há uma tendência cada vez maior de incluir técnicas quantitativas e análises estatísticas nos estudos etnodirigidos, tendência essa mais fortemente sentida a partir da década de 1990 . Todavia, os métodos quantitativos e as análises estatísticas não substituem uma coleta cuidadosa e criteriosa dos dados, pois são apenas ferramentas. De maneira muito pertinente, Bernard (1995) ressalta para a importância de manter os conceitos de quantificação e ciência separados, lembrando que qualquer ciência baseia-se primeiramente em dados qualitativos.

\section{Problemas na coleta de dados}

Há muitas maneiras de obter informações junto a uma comunidade sobre as plantas usadas para fins terapêuticos, mas é necessário, antes de iniciar qualquer pesquisa, conhecer as pessoas, como elas vivem, a sua cultura e a sua organização social. Dispomos hoje de muitos manuais de pesquisa (Martin, 1995; Alexiades, 1996), inclusive em língua portuguesa (Albuquerque; Lucena, 2004), que são direcionamentos seguros para fundamentar metodologicamente qualquer investigação.
Nem sempre é possível obter toda a informação de um grupo sobre as suas práticas relativas a doença e cura, pois muitas são revestidas de fortes valores culturais, como em algumas tribos indígenas ou comunidades afrobrasileiras, para as quais determinadas informações são segredos guardados ciosamente e transmitidos apenas para os sucessores diretos (Albuquerque, 2001, 2006b; Ikuta, 2001). Não se trata, muitas vezes, de negar simplesmente o acesso do pesquisador aos dados que ele busca, mas de preservar um conhecimento revestido de valores culturais, sociais e até mesmo sentimentais. No momento do trabalho de campo, o pesquisador deve procurar se colocar no lugar do entrevistado, pois assim é possível compreender por quê muitos segredos não podem ser revelados (Amorozo, 1996). O pesquisador em etnobotânica e/ou etnofarmacologia é essencialmente um extrator e compilador de informações. Por isso, uma posição essencial para este pesquisador é o respeito pelas instituições sociais locais.

Para um iniciante-observador na pesquisa etnodirigida, o processo de catalogação das informações pode parecer uma tarefa fácil, na qual se transcreve para uma caderneta os dados fornecidos durante uma entrevista. Mais do que uma simples técnica científica, entrevistar é uma arte, pouco compreendida por quem está à margem desses estudos. Muitos problemas nas publicações, que afetam sua qualidade e reprodutibilidade, resultam de: desconhecimento de técnicas que facilitam o contato inicial com os entrevistados, perguntas mal elaboradas, seleção inadequada ou equivocada dos entrevistados, amostragem insuficiente, desconhecimento teórico para suportar decisões metodológicas. Aliado a estes fatores, há inúmeras técnicas de coleta de dados que podem substituir ou complementar as tradicionais entrevistas, cuja seleção depende dos objetivos propostos (ver, por exemplo, Albuquerque; Lucena, 2004). Os questionários, ferramentas muito comuns em levantamentos etnofarmacológicos, podem não ser tão eficientes em função do contexto cultural (ver Edwards et al., 2005). Com base nisso, Edwards et al. (2005) ressaltam que os questionários podem inibir os informantes por se tratarem de um instrumento muito formal, por conterem questões de difícil entendimento por parte dos entrevistados (considerando os contrastes culturais e ambientais), bem como a sua, dependendo do foco da pesquisa, provocar hostilidade (ver também Albuquerque et al., 2007).

Muitos outros cuidados são necessários nesse tipo de investigação. Por exemplo, o simbólico está presente nas práticas de diferentes grupos étnicos, associado à crença em divindades e na realização de rituais que são necessários para a preparação de medicamentos e outras formas de cura (Albuquerque, 2006b). Nesse sentido, a cosmovisão de um povo dita a forma com que as pessoas se relacionam com as plantas ou animais, bem como sua a percepção sobre o mundo e sobre as relações entre as partes que o compõe. É tarefa do pesquisador decodificar as informações recebidas, procurando aproximar as 
Quadro 2. Roteiro para avaliação de trabalhos científicos na área de etnobotânica e etnofarmacologia. Baseado nos critérios apresentados por Lima et al. (1999) e Castro (2002) para artigos científicos, dissertações e teses, e McClatchey (2006) para os problemas encontrados nas publicações em etnobotânica.

\author{
Definição do problema e dos objetivos \\ A) A área ou tema de estudo estão bem delimitadas? \\ B) O problema da pesquisa está bem definido e bem delimitado? \\ C) Os objetivos são claros e bem formulados?
}

Introdução e fundamentação teórica

A) Apresenta os elementos teóricos necessários para embasar o problema?

B) Apresenta dados consistentes e atualizados sobre as variáveis, os elementos importantes e os métodos para o estudo do problema?

C) Enuncia as hipóteses? São comentadas a luz dos achados na literatura?

D) Estabelece relações entre as diferentes variáveis?

E) A redação do texto é clara, concisa, inter-relacionada, estruturada e organizada?

\title{
Métodos
}

A) Caracteriza o local de trabalho? Se for o caso: tamanho e status da área, infra-estrutura, problemas ambientais, o ambiente físico e biológico (vegetação), agricultura, extrativismo, turismo, demografia etc.

B) O delineamento amostral está de acordo com os objetivos?

C) Define a população?

D) As comunidades estudadas estão profundamente caracterizadas? Etnia, costumes, história de ocupação do espaço, traços marcantes da cultura, tamanho da população, religião, saúde e educação, dentre outros.

E) Os informantes/entrevistados estão detalhadamente descritos?

F) Descreve a unidade de observação e amostragem?

G) Descreve os critérios utilizados para a seleção da amostra?

H) Explicita se obteve autorização formal dos entrevistados para realização da pesquisa?

I) Define o tamanho da amostra e o tipo de amostragem?

J) Descreve as informações a serem colhidas?

K) Descreve os procedimentos utilizados para coleta de dados?

L) As técnicas e os equipamentos estão bem descritas e são adequadas?

M) Os métodos estatísticos para as análises estão explicitados e são adequados?

N) As plantas e/ou animais foram coletados e identificados pelos procedimentos usuais? A identificação por dicionários ou listas, em raras ocasiões, é aceitável.

O) Para estudos com vistas à descoberta de novos fármacos, um item importante é uma boa descrição etnográfica dos sistemas terapêuticos e dos conceitos de saúde e doença. Também é fundamental compreender, além dos nomes das plantas e dos seus correspondentes botânicos, os nomes das doenças/sintomas e seus correspondentes em termos de sistemas médicos ocidentais/urbanos

\section{Resultados}

A) Estão apresentados de maneira clara e concisa?

B) As tabelas, esquemas, gráficos e figuras, são apresentados de maneira clara e concisa?

C) Todas as ilustrações, tabelas e gráficas são necessários?

D) As espécies estão corretamente citadas?

E) As espécies citadas foram adequadamente amostradas com o registo e depósito de material testemunho em herbários ou coleções apropriadas?

\section{Discussão}

A) O autor estabelece comparações com os dados da literatura?

B) Os resultados são discutidos de maneira clara em relação aos objetivos propostos?

C) Os resultados comprovam ou anulam as hipóteses propostas?

D) Se o trabalho apresentar uma lista de plantas e/ou animais usados como medicinais, existe uma discussão contextualizada de seus usos?

E) As conclusões, quando presentes, contribuem para um melhor entendimento das relações entre pessoas e recursos medicinais?

\section{Bibliografia}

A) As referências bibliográficas citadas estão redigidas conforme as normas?

B) A redação está correta?

C) Reflete o empenho dos pesquisadores por enriquecer o trabalho? 
Dados complementares

A) O título está adequado?

B) Atende aos requisitos legais no caso de pesquisas com seres humanos?

C) Cronograma, quando for o caso, está bem descrito com as etapas factíveis no tempo previsto?

D) A linguagem é aceitável?

observações emicistas às explicações eticistas. Por exemplo, há várias formas de se conceber e de tratar as doenças, implicando no desafio de aceitar as diferenças culturais e lingüísticas, assumindo o compromisso de um esforço de interpretação (Amorozo, 1996), que nem sempre resulta satisfatório. Além disso, se o estudo estiver voltado para a bioprospecção, algumas informações são muito importantes e, em alguns casos, indispensáveis (Quadro 3). Bermúdez et al. (2005) argumentam ainda que os estudos etnobotânicos com plantas medicinais deveriam envolver: registro dos dados sobre as plantas medicinais relacionadas com a comunidade estudada com a coleta de todas as informações possíveis (ver quadro 3); análise quantitativa da importância cultural ou nível de uso das diferentes espécies; determinação do padrão de variação do conhecimento tradicional e sua relação com fatores sociais que o afetam; análise das estratégias empregadas pela população para o aproveitamento das plantas medicinais; determinação da abundância, distribuição e diversidade das plantas medicinais usadas; avaliação do impacto extrativista sobre a estrutura e diversidade dos ecossistemas naturais; desenho de projetos de aproveitamento sustentável ou estratégias de conservação dos recursos levando em consideração os saberes e práticas tradicionais; desenvolvimento de mecanismos para o reconhecimento público dos direitos intelectuais das populações estudadas com o desenvolvimento de estratégias para compensá-las por sua participação nas investigações. Obviamente, essa seria uma proposta ideal que depende de um esforço pelo menos interdisciplinar para o seu sucesso.

\section{Problemas na apresentação e análise dos dados}

Uma das dificuldades nas pesquisas etnodirigidas é a imprecisão das informações publicadas. Waller (1993) exemplifica com o caso das "inflamações", pois estas podem ocorrer em praticamente todo o corpo. As causas podem ser diversas: trauma, infecções, alergia ou asma. A literatura etnobotânica, por exemplo, tem muitas referências para atividade antiinflamatória com pouco ou nenhum suporte etiológico que subsidie decisões futuras sobre os modelos farmacológicos mais apropriados. Dessa maneira, o simples termo "atividade antiinflamatória" não dá suporte para a seleção do modelo para avaliação farmacológica, sendo necessário descrições detalhadas do distúrbio (Waller, 1993). Para um estudo etnodirigido ser considerado útil, algumas informações a respeito da etiologia da doença, preparação da planta, modo de administração, objetivos terapêuticos e outros detalhes que subsidiam os pesquisadores a entender a cultura local devem ser adequadamente coletados e claramente apresentados (Etkin, 2001) (ver novamente o quadro 3).

Há várias técnicas e métodos (ver Albuquerque; Lucena, 2004) que podem reduzir a subjetividade na apresentação das informações sobre plantas medicinais e a realização de testes farmacológicos, ao mesmo tempo em que oferecem um parâmetro para tomada de decisões sobre qual planta é ou não merecedora de um estudo mais aprofundado. Por exemplo, Canales et al. (2005) utilizando as técnicas tradicionais em estudos etnobotânicos (lista livre e entrevistas semi-estruturadas), tiveram como objetivo coletar informações sobre os usos de plantas por uma comunidade em Puebla (México) e ao mesmo tempo selecionar plantas para estudos de atividade biológica usando o fator de consenso dos informantes. $\mathrm{O}$ fator de consenso dos informantes é uma técnica quantitativa que permite indicar plantas e/ou doenças que gozam de maior consenso entre as pessoas, bastante usada por diferentes pesquisadores (Amiguet et al., 2005; Gazzaneo et al., 2005; Almeida et al., 2006; Alves; Rosa, 2006). Na investigação de Canales et al. (2005), os informantes entraram em grande consenso ao indicar plantas para tratar problemas com possível origem bacteriana. Dessa forma, os pesquisadores selecionaram 16 espécies para teste de atividade antibacteriana, sendo que $75 \%$ destas mostraram a referida atividade. Técnicas quantitativas também têm sido usadas para selecionar plantas com atividade hipoglicemiante (Leduc et al., 2006; AndradeCetto et al., 2006; Funke; Melzig, 2006).

Utilizando a lógica do consenso dos informantes, Berlin (1999) analisou a variação léxica nos nomes atribuídos as plantas medicinais entre 14 municípios Tzotzil e Tzeltal, no México. O pressuposto era de que as espécies com maior consenso no seu uso e, portanto, que apresentavam uma menor variação léxica, seriam as espécies com maior potencial farmacológico, em oposição às espécies com usos mais amplos para muitas condições de saúde, que apresentavam uma maior variação léxica.

\section{Ausência de integração entre resgate de informações, teoria e replicabilidade}

Apesar da grande necessidade de estudos etnodirigidos para permitir a recuperação e a conservação de conhecimentos sobre plantas medicinais nativas no Brasil, tendo em vista a seleção de plantas para estudos farmacológicos, toxicológicos e fitoquímicos (Di-Stasi, 2005), existe quase que uma completa ausência do uso ou desenvolvimento de teorias para abarcar os fenômenos 
Quadro 3. Tipo de informação que necessita ser coletada em estudos etnodirigidos. Fonte: Berlin e Berlin (2005).

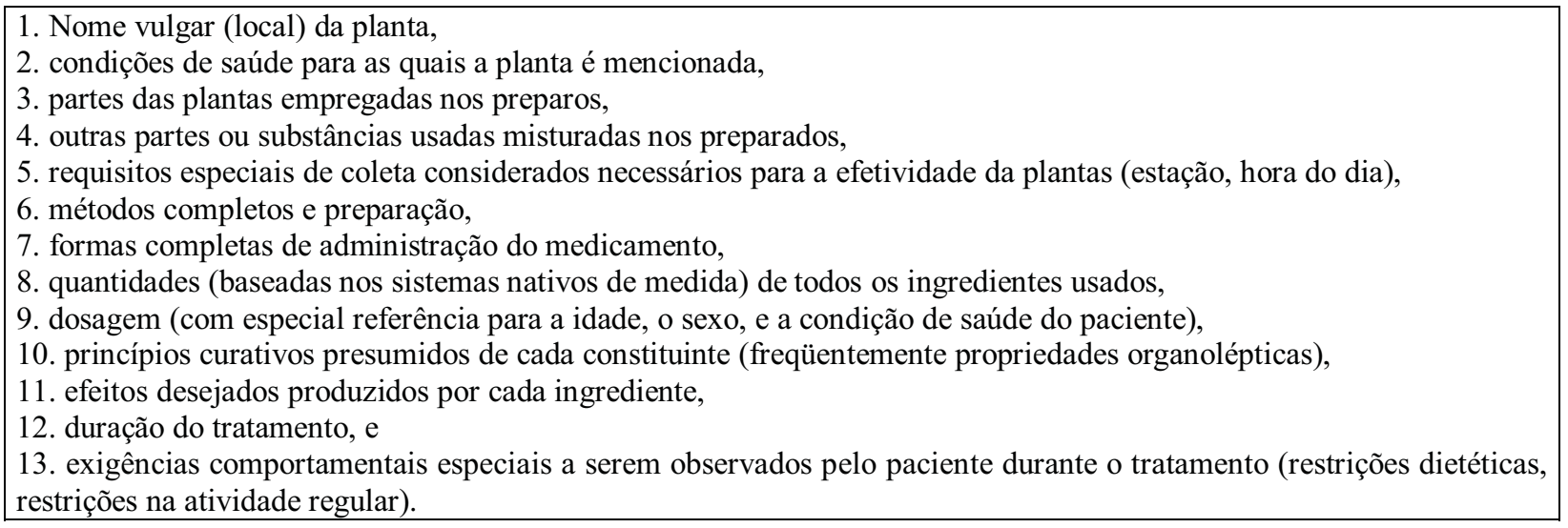

observados e fazer avançar ainda mais rápido uma teoria da relação entre pessoas e os recursos naturais usados em sua medicina. A construção ou uso de uma teoria é essencial para qualquer tipo de abordagem científica que se pretenda capaz de responder questões e prever fenômenos. Há vários fatores que afetam o conhecimento das pessoas sobre as plantas medicinais que utilizam: ecológicos, econômicos, sociais e culturais e que agem de diferentes formas em diferentes culturas. Do mesmo modo, existe quase que uma completa ausência de replicabilidade nos estudos com diferentes grupos e formações vegetais, mesmo entre estudos que adotam uma abordagem comparativista. Tal replicabilidade seria desejável para possibilitar testes de hipóteses com métodos semelhantes, em diferentes contextos, o que permitiria que compreendamos melhor o contexto das relações entre as pessoas e as plantas e/ou animais que usam em sua medicina. Neste sentido, é preciso tornar estes estudos etnodirigidos mais preditivos, o que implica em revestir de maior rigor as pesquisas.

As questões éticas que permeiam os estudos etnobotânicos e etnofarmacológicos

Os estudos etnodirigidos têm como objetivo, como refere Etkin (2001) para a etnofarmacologia, principalmente: a bioprospecção e ao avanço da ciência farmacêutica, com a descoberta de novos fármacos e a sua inclusão na farmacopéia biomédica; a conservação e preservação da biodiversidade; promover o uso local das plantas em combinação com os fármacos já conhecidos e outras tecnologias biomédicas; bem como fazer uso do conhecimento das comunidades locais respeitando a sua propriedade intelectual. Parece-nos que hoje esses objetivos são um tanto contraditórios, pois a bioprospecção e o avanço da ciência farmacêutica são atividades movidas por um mecanismo subjacente capitalista e ligado à acumulação individual de lucros, ou restrita à pequenos grupos ou grandes corporações. Já a conservação da biodiversidade, nem sempre. Muito menos o respeito à propriedade intelectual local, que muitas vezes é difusa e calcada em uma lógica coletiva e não individual. Isso pode parecer um pouco partidarista, mas os sistemas de proteção individual do conhecimento (e por consequência do retorno de benefícios financeiros de pesquisas ligadas à bioprospecção e à ciência farmacêutica) parecem não beneficiar de fato as populações locais através dos mecanismos que são utilizados hoje (Funari; Ferro, 2005).

Uma questão recorrente na atualidade remete à proteção do conhecimento local sobre plantas e animais medicinais. Não há um consenso, muito embora vários pesquisadores já adotem determinados procedimentos durante suas pesquisas, principalmente na divulgação dos resultados. No caso de plantas medicinais, por exemplo, há pesquisadores que não divulgam plantas interessantes encontradas em seus estudos até que se construa um instrumento que garanta à comunidade, detentora original do conhecimento, os devidos direitos, caso a planta venha originar um medicamento (cf. Rodrigues; Carlini, 2003a, b). Segundo Platiau e Varella (2004), o Brasil foi um dos países pioneiros nas negociações internacionais em favor da soberania nacional sobre os recursos genéticos, mas criou uma estrutura ineficaz e burocrática para a gestão de pedidos de acesso através da Medida Provisória n. 2.186-16, de 23 de agosto de 2001, regulamentada pelo Decreto n. 3.945, de 23 de setembro de 2001. No Brasil, a proteção do conhecimento tradicional vem sendo amplamente discutida em função desta Medida que visa regulamentar o acesso ao conhecimento tradicional associado a recursos genéticos, implicando em acompanhamento de procedimentos e autorizações de acesso, cabendo este papel ao CGEN (Conselho de Gestão do Patrimônio Genético), órgão ligado ao Ministério do Meio Ambiente.

Todavia, há vários problemas práticos, não previstos, que precisam ser solucionados para não impedir o desenvolvimento do Brasil em pesquisas nessa área. Há 
Quadro 4. Modelo de Termo de Consentimento Livre Esclarecido.

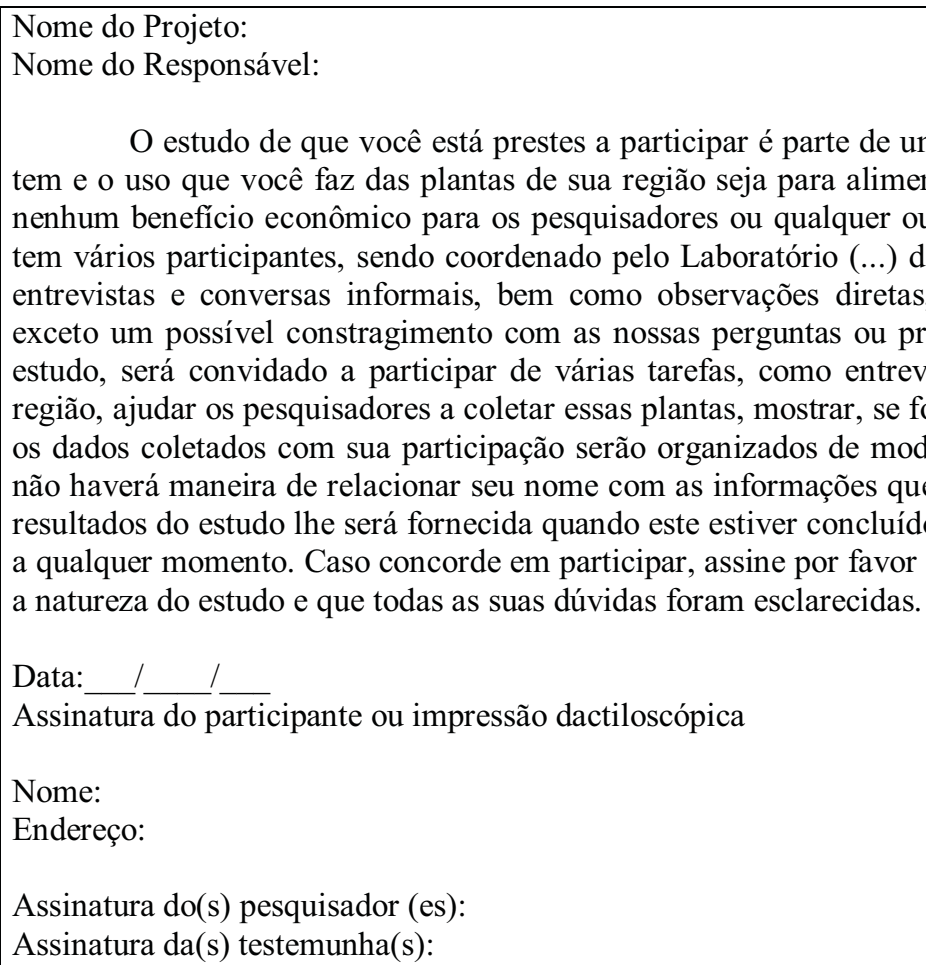
a natureza do estudo e que todas as suas dúvidas foram esclarecidas.

Data:

Assinatura do participante ou impressão dactiloscópica

Nome:

Endereço:

Assinatura do(s) pesquisador (es):

Assinatura da(s) testemunha(s):

O estudo de que você está prestes a participar é parte de uma série de estudos sobre o conhecimento que você tem e o uso que você faz das plantas de sua região seja para alimentação, construção, lenha, medicina etc., e não visa nenhum benefício econômico para os pesquisadores ou qualquer outra pessoa ou instituição. É um estudo amplo, que tem vários participantes, sendo coordenado pelo Laboratório (...) da Universidade (...). $\mathrm{O}$ estudo emprega técnicas de entrevistas e conversas informais, bem como observações diretas, sem riscos de causar prejuízo aos participantes, exceto um possível constragimento com as nossas perguntas ou presença. Caso você concorde em tomar parte nesse estudo, será convidado a participar de várias tarefas, como entrevistas, listar as plantas que você conhece e usa da região, ajudar os pesquisadores a coletar essas plantas, mostrar, se for o caso, como você as usa no seu dia a dia. Todos os dados coletados com sua participação serão organizados de modo a proteger a sua identidade. Concluído o estudo, não haverá maneira de relacionar seu nome com as informações que você nos forneceu. Qualquer informação sobre os resultados do estudo lhe será fornecida quando este estiver concluído. Você tem total liberdade para se retirar do estudo a qualquer momento. Caso concorde em participar, assine por favor seu nome abaixo, indicando que leu e compreendeu

um consenso de que deve haver a repartição de benefícios, com a realização de contratos (para os quais ainda não há unanimidade) de utilização dos recursos-alvo. O tema da bioprospecção encontra-se em forte discussão no Brasil, pois os pesquisadores são unânimes quanto à repartição de benefícios, mas pedem justiça e flexibilidade para que as leis não inviabilizem o progresso da pesquisa. Além disso, a idéia de direitos de propriedade ainda necessita ampla discussão. McClatchey (2006) ressalta que, subjacente ao conceito de direito de propriedade, considerando "a melhor das intenções paternalistas", existe a idéia de que todas as culturas o entendem da mesma forma, o que representa um grande equívoco. Há pessoas, em algumas comunidades, que ficam apreensivas com a idéia de "possuir o conhecimento", enquanto outras fortemente defendem seus direitos de propriedade de conhecimento (McClatchey 2005).

As medidas governamentais criadas para regulamentar $\mathrm{o}$ acesso à biodiversidade $\mathrm{e}$ aos conhecimentos tradicionais associados a ela também visam estabelecer mecanismos de repartição de benefícios gerados por pesquisas com esta natureza. Entretanto, corremos o risco de recair novamente na estrutura burocrática ineficaz. Com relação ao retorno de benefícios oriundos das pesquisas etnodirigidas, este deve ser encarado primeiramente como um pressuposto ético e moral do próprio pesquisador, e não apenas como uma necessidade legal. Elisabetsky (2003) sugere que a repartição de benefícios pode ocorrer tanto através de compensação monetária (por exemplo, de imediato quando há um pagamento pelos dias de trabalho do informante ou um pagamento por amostras de plantas coletadas; ou através de repasse de percentuais dos royalties gerados por aquele conhecimento) ou por formas não monetárias de repartição de benefícios, como contribuições à economia local/municipal, melhorias na capacitação das populações locais quanto ao uso sustentado de recursos naturais/genéticos e capacitação administrativa, intercâmbio de equipes e treinamentos, transferência de tecnologia, e aumento da capacidade científica local, com a participação de especialistas locais.

Independente das discussões acima, as pesquisas que envolvem acesso ao conhecimento das pessoas devem também respeitar os preceitos da Resolução no. 196, de 10/10/1996, do Conselho Nacional de Saúde que define pesquisa envolvendo seres humanos como aquela que "envolva o ser humano, de forma direta ou indireta, em sua totalidade ou partes dele, incluindo o manejo de informações ou materiais". Assim, segundo tal resolução, o projeto de pesquisa deve ser previamente aprovado pelo Comitê de Ética em Pesquisa (CEP) da instituição, contando com o consentimento livre e esclarecido das pessoas que serão o alvo do trabalho. No quadro 4 apresentamos um modelo de Termo de Consentimento Livre Esclarecido para pesquisa que não envolve retorno econômico. É necessário ressaltar que as informações sugeridas no quadro 4 devem ser adequadas às diferentes situações de estudo e aos grupos estudados, de maneira a assegurar a sua clareza e sua compreensão pelas pessoas que participarão do estudo fornecendo as 
informações etnobotânicas. Quase sempre é necessário efetuar contatos prévios com lideranças comunitárias, bem como organizar reuniões e discussões em grupos e outras formas de comunicação.

\section{CONCLUSÃO}

Para se ter uma idéia e uma síntese do que comentamos, recentemente um editorial do Journal of Ethnopharmacology (Verpoorte et al., 2006), um dos mais importantes periódicos na área, faz recomendações aos autores para minimizar a rejeição de seus trabalhos, no sentido de evitar a ausência de: informações etnográficas do estudo ou sobre os métodos usados; referencial teórico que constitui a base do estudo; de informações sobre o diagnóstico de doenças e práticas relativas ao uso médico específico de uma planta; informações sobre a proteção e os direitos de povos indígenas ou governo local (ver Pieroni, 2006). Apesar de Pieroni (2006) criticar a redação de alguns itens acima, como pouco claros e confusos, é certo que o texto comentado já reflete uma tendência de aumentar o rigor na aceitação de trabalhos etnobotânicos e etnofarmacológicos, como também pode ser constatado em outro recente editorial do Economic Botany (Moerman, 2005), periódico de referência em etnobotânica.

Mais importante do que as considerações sobre o rigor metodológico das pesquisas etnodirigidas são as considerações sobre as implicações éticas da pesquisa, no contexto das discussões atuais sobre acesso a conhecimentos associados à biodiversidade brasileira $\mathrm{e}$ retorno de resultados das pesquisas. A legislação brasileira atual ainda é limitada para regulamentar tais questões. É preciso que o pesquisador constantemente se questione sobre os benefícios do seu fazer acadêmico ou, segundo Posey e Dutfield (1996), pesquisa-se o conhecimento de quem para benefício de quem?

Vivencia-se um momento propício e produtivo para a pesquisa científica que envolve a aplicação de conhecimentos locais sobre o uso de plantas e animais medicinais. Deixou-se para trás a época em que esse saber era subestimado, iniciando hoje uma era de cooperação de saberes. Todavia, para responder aos questionamentos e necessidades sociais, é forçoso avançar as abordagens para produzir uma ciência que venha realmente atender aos anseios das comunidades locais, da sociedade como um todo e da própria comunidade científica. $\mathrm{E}$ isto não é uma tarefa fácil, com uma receita pronta. Neste artigo colocamos algumas questões na esperança de refletirmos sobre as nossas necessidades e no cenário atual que exige rápidas mudanças e avanços. "Um dos objetivos mais importantes da bioprospecção da biodiversidade é ajudar a conservar a grande diversidade de línguas, culturas, povos e outros organismos que habitam a terra. Um dos desafios que antropólogos, etnobotânicos, médicos empreendedores, e profissionais do desenvolvimento enfrentam é utilizar criativamente a bioprospecção da biodiversidade como uma das muitas ferramentas para manter e manejar a fértil, mas frágil, diversidade de pessoas, plantas, culturas e ecossistemas que estão sob ameaça constante de extinção" (Moran et al., 2001).

\section{AGRADECIMENTOS}

Os autores agradecem a Ernani Machado de Freitas Lins Neto, do Laboratório de Etnobotânica Aplicada (LEA-UFRPE), pelo apoio na obtenção de literatura e discussão de algumas idéias. Ao $\mathrm{CNPq}$ pelo auxílio financeiro (Produtividade em Pesquisa) para U.P. Albuquerque.

\section{REFERÊNCIAS}

Albuquerque UP 2001. The use of medicinal plants by the cultural descendants of African people in Brazil. Acta Farm Bonaerense 20: 139-144.

Albuquerque UP, Lucena RFP (Orgs.) 2004. Métodos e técnicas na pesquisa etnobotânica. Recife: Livro Rápido, NUPEEA.

Albuquerque UP 2005. Introdução à etnobotânica. 2.ed. Rio de Janeiro: Editora Interciência.

Albuquerque UP, Lucena RFP 2005. Can apparency affect the use of plants by local people in tropical forests? Interciencia 30: 506-511.

Albuquerque UP, 2006a. Re-examining hypotheses concerning the use and knowledge of medicinal plants: a study in the Caatinga vegetation of NE Brazil. J. Ethnobiol Ethnomedicine 2: 30.

Albuquerque UP 2006b. Folhas sagradas - as plantas litúrgicas e medicinais nos cultos afro-brasileiros. 2.ed. Recife: NUPEEA.

Albuquerque UP, Monteiro JM, Ramos MA, Amorim ELC 2007. Medicinal and magic plants from a public market in northeastern Brazil. J. Ethnopharmacol doi:10.1016/ j.jep.2006.09.010.

Alexiades M 1996. Selected guidelines for ethnobotanical research: a field manual. Nova York: New York Botanical Garden.

Almeida CFCBR, Albuquerque UP 2002. Uso e conservação de plantas e animais medicinais no estado de Pernambuco (Nordeste do Brasil): um estudo de caso. Interciencia 27: $276-285$

Almeida CFCBR, Silva TCL, Amorim ELC, Maia MBS, Albuquerque UP 2005. Life strategy and chemical composition as predictors of the selection of medicinal plants from the Caatinga (Northeast Brazil). J Arid Environ 62: 127-142.

Almeida CFCBR, Amorim ELC, Albuquerque UP, Maia MBS 2006. Medicinal plants popularly used in the Xingo region - a semi-arid location in Northeastern Brazil. $J$ Ethnobiol Ethnomedicine 2: 15.

Alves AGC, Albuquerque UP 2005. Exorcizando termos em etnobiologia e etnoecologia. In: Alves AGC, Lucena RFP, Albuquerque UP (org.) Atualidades em etnobiologia e etnoecologia: volume 2 Recife: Nupeea; Sociedade Brasileira de Etnobiologia e Etnoecologia.

Alves RR, Rosa IL 2006. From cnidarians to mammals: The use 
of animals as remedies in fishing communities in $\mathrm{NE}$ Brazil J Ethnopharmacol 107: 259-276.

Amiguet VT, Arnason JT, Maquin P, Cal V, Vindas PS, Poveda L 2005. A consensus ethnobotany of the Q'eqchi' Maya of southern Belize. Econ Bot 59: 29-42.

Amorozo MCM 1996. A abordagem etnobotânica na pesquisa de plantas medicinais. In: Di-Stasi LC (Org.) Plantas medicinais: arte e ciência. São Paulo: Unesp, p.4768.

Andrade-Cetto A, Becerra-Jiménez J, Martinez-Zurita E, Ortega-Larrocea P, Heinrich M 2006. DiseaseConsensus Index as a tool of selecting potential hypoglycemic plants in Chikindzonot, Yucatán, México. J Ethnopharmacol 107: 199-204.

Balick MJ, Cox PA 1996. Plants, people and culture: the science of ethnobotany. New York: HPHLP.

Berkes F 1999. Sacred ecology. Philadelphia: Taylor and Francis.

Berlin B 1999. Lexical reflections on the cultural importance of medicinal plants among Tzotzil and Tzeltal Maya. In: Gragson TL, Blount BG. Ethnoecology: knowledge, resources and rights. Athens: The University of Georgia Press, p.12-23.

Berlin EA, Berlin B 2005. Some field methods in medical ethnobiology. Field Meth 17: 235-268.

Bermúdez A, Oliveira-Miranda MA, Velázquez D 2005. La investigación etnobotánica sobre plantas medicinales: una revisión de sus objetivos y enfoques actuales. Interciencia 30: 453-459.

Bernard HR 1995. Research methods in anthropology: qualitative and quantitative approaches. 2.ed. London: Altamira press.

Bruhn JG, Holmstet B 1980. Ethnopharmacology: objectives, principles and perspectives. In: Beal JL, Reinhard E (eds.) Natural products as medicinal agents Planta Medica Supplement, 526p.

Calderón AI, Angerhofer CK, Pezzuto JM 2000. Forest plot as a tool to demonstrate the pharmaceutical potential of plants in a tropical forest of Panama. Econ Bot 54: 278-294.

Canales M, Hernández T, Caballero J, Romo de Vivar A, Avila G, Duran A, Lira R 2005. Informant consensus factor and antibacterial activity of the medicinal plants used by the people of San Rafael Coxcatlán, Puebla, México J Ethnopharmacol 97: 429-439.

Carrai V, Borgognini-Tarli SM, Huffman MA, Bardi M 2003. Increase in tannin consumption by sifaka (Propithecus verreauxi verreauxi) females during the birth season: a case for self medication in prosimians? Primates 44: 61-66.

Castro AA 2002. Avaliação crítica da literatura: roteiro para a sessão de artigos de revista. Maceió: AAC. Disponível em http://www.metodologia.org.

Daudt R, von Poser GL, Neves G, Rates SM 2000 Screening for the antidepressant activity of some species of Hypericum from South Brazil. Phytother Res 14: 344346.

Desmarchelier C, Lisboa Romão R, Coussio J, Ciccia G 1999. Antioxidant and free radical scavenging activities in extracts from medicinal trees used in the 'Caatinga' region in northeastern Brazil J Ethnopharmacol 67: 69-77.

Di-Stasi LC 2005. An integrated approach to identification and conservation of medicinal plants in the tropical forest - a Brazilian experience. Plant Genetic Resources 3: 199 a 205.

Diegues AC, Arruda RSV (orgs.) 2001. Saberes tradicionais e biodiversidade no Brasil. Brasília: Ministério do Meio Ambiente; São Paulo: USP

Diehl MS, Kamanzi Atindehou K, Téré H, Betschart B 2004. Prospect for anthelminthic plants in the Ivory Coast using ethnobotanical criteria. J Ethnopharmacol 95: 277-284.

Duarte MCT, Figueira GM, Pereira B, Magalhães PM, Delarmelina C 2004. Atividade antimicrobina de extratos hidroalcoólicos de espécies da coleção de plantas medicinais CPQBA/UNICAMP. Rev Bras Farmacogn 14(Supl. 1): 6-8.

Edwards S, Nebel S, Heinrich M 2005. Questionnaire surveys: Methodological and epistemological problems for field-based ethnopharmacologists. $J$ Ethnopharmacol 100: $30-36$

Elisabetsky E 2003. Direitos de propriedade intelectual e distribuição equitativa de benefícios no contexto de inovação tecnológica. In: Anais do I Simpósio de Etnobiologia e Etnoecologia da região Sul: aspectos humanos da biodiversidade. Florianópolis: SBEE, p. 170-174.

Estomba D, Ladio A, Lozada M 2006. Medicinal wild plant knowledge and gathering patterns in a Mapuche community from North-western Patagonia. J Ethnopharmacol 103: 109-119.

Etkin NL 2001. Perspectives in ethnopharmacology: forging a closer link between bioscience and traditional empirical knowledge. J Ethnopharmacol 76: 177182.

Funari CS, Ferro VO 2005. Uso ético da biodiversidade brasileira: necessidade e oportunidade. Rev Bras Farmacogn 15: 178-182.

Funke I, Melzig MF 2006. Traditionally used plants in diabetes therapy - phytotherapeutics as inhibitors of $\alpha$-amylase activity. Rev Bras Farmacogn 16: 1-5.

Gadgil M, Berkes F, Folke C 1993. Indigenous knowledge for biodiversity conservation. Ambio 22: 151-156.

Gazzaneo LR, Lucena RFP, Albuquerque UP 2005. Knowledge and use of medicinal plants by local specialists in a region of Atlantic Forest in the state of Pernambuco (Northeastern Brazil). J Ethnobiol Ethnomedicine 1: 9.

Hiruma-Lima CA, Santos LC, Kushima H, Pellizzon CH, Silveira GG, Vasconcelos PCP, Vilegas W, Souza Brito ARM 2006. Qualea grandiflora, a Brazilian "Cerrado" medicinal plant presents an important antiulcer activity. J Ethnopharmacol 104: 207-214.

Huffman MA 2001. Self-medicative behavior in the African Great Apes: an evolutionary perspective into the origins of the human traditional medicine. BioScience 51: 651-661.

Huffman MA 2003. Animal self-medication and ethnomedicine: exploration and exploitation of the medical properties of plants. P Nutr Soc 63: 371-381.

Ikuta ARY 2001. Práticas fitotécnicas de uma comunidade indígena mbyá guarani, Varzinha, Rio Grande do Sul: da roça ao artezanato. Porto Alegre, 307 p. Tese de doutorado - Agronomia (Fitotecnia), Universidade Federal do Rio Grande do Sul. 
Jorge RM, Leite JPV, Oliveira AB, Tagliati CA 2004. Evaluation of antinociceptive, anti-inflammatory and antiulcerogenic activities of Maytenus ilicifolia. $J$ Ethnopharmacol 94: 93-100.

Khafagi IK, Dewedar A. 2000. The efficiency of random versus ethno-directed research in the evaluation of Sinai medicinal plants for bioactive compounds. $J$ Ethnopharmacol 71: 365-376.

Krief S, Martin M, Grellier P, Kasenene J, Sévenet T 2004. Novel antimalarial compounds isolated in a survey of self-medicative behavior of wild chimpanzees in Uganda. Antimicrob Agents Ch 48: 3196-3199.

Leduc C, Coonishish J, Haddad P, Cuerrier A 2006. Plants used by the Cree Nation of Eeyou Istchee (Quebec, Canada) for the treatment of diabetes: A novel approach in quantitative ethnobotany. $J$ Ethnopharmacol 105: 5563.

Lima CP, Jeckel-Neto EA, Baracat EC, Rudge MVC, Arkader J 1999. Analisando teses e dissertações. Femina 27 805-808.

Lima MRF, Ximenes CPA, Luna JS, Sant'Ana AEG 2006. The antibiotic activity of some Brazilian medicinal plants. Rev Bras Farmacogn 16: 300-306.

Macedo M, Ferreira AR 2004. Plantas hipoglicemiantes utilizadas por comunidades tradicionais na Bacia do Alto Paraguai e Vale do Guaporé, Mato Grosso-Brasil. Rev Bras Farmacogn 14(Supl. 1): 45-47.

Maciel MAM, Pinto AC, Veiga Jr VF, Grynberg NF 2002. Plantas medicinais: a necessidade de estudos multidisciplinares. Quim Nova 25: 429-438.

Martin GJ 1995. Ethnobotany: a people and plants conservation manual. London: Chapman \& Hall.

McClatchey W 2005. Exorcing misleading terms from ethnobotany. Ethnobotany Research \& Applications 3: $1-4$

McClatchey W 2006. Improving quality of international ethnobotany research and publications. Ethnobotany Research \& Applications 4: 1-9.

Mendonça-Filho RFW, Menezes FS 2003. Estudo da utilização de plantas medicinais pela população da Ilha GrandeRJ. Rev Bras Farmacogn 13(Supl): 55-58.

Moerman DE 2005. An open letter to SEB members, and readers of Economic Botany, from the editor in chief. Econ Bot 59: 309-310.

Monteiro JM, Albuquerque UP, Lins Neto EMF, Araujo EL, Amorim ELC 2006. Use patterns and knowledge of medicinal species among two rural communities in Brazil's semi-arid northeastern region. $J$ Ethnopharmacol 105: 173-186.

Moran K, King SR, Carlson TJ 2001. Biodiversity prospecting lessons and prospects. Annu Rev Anthropol 30: 505526.

Morais SM, Dantas JDP, Silva ARA, Magalhães EF 2005. Plantas medicinais usadas pelos índios Tapebas do Ceará. Rev Bras Farmacogn 15: 169-177.

Nunes GP, Silva MF, Resende UM, Siqueira JM 2003. Plantas medicinais comercializadas por raizeiros no Centro de Campo Grande, Mato Grosso do Sul. Rev Bras Farmacogn 13: 83-92.

Pieroni A 2006. Journal of Ethnobiology and Ethnomedicine: achievements and perspectives. $J$ Ethnobiol Ethnomedicine 2: 10

Platiau AFB, Varella MD 2004. Diversidade biológica e conhecimentos tradicionais. Coleção Direito Ambiental. Belo Horizonte: Del Rey.

Posey DA, Dutfield G 1996. Beyond intellectual property: Toward traditional resource rights for indigenous peoples and local communities. Ottawa: International Development Research Center.

Rodrigues E, Carlini E 2003a. Levantamento etnofarmacológico realizado entre um grupo de quilombolas do Brasil. Arq Bras Fitomed Cient 1: 80-87.

Rodrigues E, Carlini E 2003b. Possíveis efeitos sobre o sistema nervoso central de plantas utilizadas por duas culturas brasileiras (quilombolas e índios). Arq Bras Fitomed Cient 1: 147-154.

Silva ML, Cechinel Filho V 2002. Plantas do gênero Bauhinia: composição química e potencial farmacológico. Quim nova 25: 449-454

Slish DF, Ueda H, Arvigo R Balick MJ 1999.Ethnobotany in the search for vasoactive herbal medicines $J$ Ethnopharmacol 66: 159-165.

Smelcerovic A, Spiteller M 2006. Phytochemical analysis of nine Hypericum L. species from Serbia and the F.Y.R. Macedonia. Pharmazie 61: 251-252.

Stepp JR, Moerman DE 2001. The importance of weeds in ethnopharmacology. J Ethnopharmacol 75: 19-23.

Stepp JR 2004. The role of weeds as sources of pharmaceuticals. J Ethnopharmacol 92: 163-166.

Vendruscolo GS, Rates SMK, Mentz LA 2005. Dados químicos e farmacológicos sobre as plantas utilizadas como medicinais pela comunidade do bairro Ponta Grossa, Porto Alegre, Rio Grande do Sul. Rev Bras Farmacogn 15: 361-372.

Verpoorte R, Houghton PJ, Heinrich M, Mukherjee PK, Hirschmann GS, van Staden J, Yesilada E 2006. Editorial. J Ethnopharmacol 103: 309-310.

Voeks RA 2004. Disturbance pharmacopoeias: medicine and myth from the humid tropics. Ann Assoc Am Geogr 94: 868-888

Waller DP 1993. Methods in ethnopharmacology. $J$ Ethnopharmacol 38: 189-195. 